\title{
Change theory and theory of change: what's the difference anyway?
}

\author{
Daniel L. Reinholz and Tessa C. Andrews ${ }^{2^{*}}$ (D)
}

\begin{abstract}
This commentary focuses on the difference between a theory of change and change theory, as it relates to systemic change projects in STEM higher education. A theory of change is project-specific and related to evaluation. It makes the underlying rationale of a project explicit, which supports planning, implementation, and assessment of the project. In addition, a theory of change is often required by funding agencies as part of grant proposals. In contrast, change theories represent theoretical and empirically grounded knowledge about how change occurs that goes beyond any one project. Ideally, a theory of change is informed by change theories. This essay describes the connections between a theory of change and change theory and provides examples of how change theory can inform a project's theory of change. Grounding projects in change theory allows change agents to draw on existing knowledge and to better contribute to our collective knowledge about how to achieve meaningful change in STEM higher education.
\end{abstract}

Keywords: Change, Reform, Theory of change, Change theory, Education reform, Undergraduate STEM

\section{Introduction}

Research in recent decades has greatly advanced our knowledge of effective and equitable teaching and learning in undergraduate science, technology, engineering, and mathematics (STEM). However, translating these discoveries into tangible reforms in undergraduate education has proven challenging. Many efforts designed to promote change in undergraduate STEM education have fallen short of their intended outcomes (Henderson, Beach, \& Finkelstein, 2011). When efforts have been successful, such as the FIRST project in biology and the New Faculty Workshops in physics, they have improved their impact over time through iterative evaluation research and revision (e.g., Henderson, 2008; Ebert-May et al. 2011; Ebert-May et al., 2015; Derting et al., 2016; Chasteen, Chattergoon, Prather, \& Hilborn, 2016; Olmstead \& Turpen, 2016). Despite some successes, on a national scale in the USA, we see that traditional instructional strategies continue to dominate in STEM classrooms (e.g., Stains et al., 2018). Furthermore, instructors who adopt evidence-based strategies often quit using them (Henderson, Dancy, \& Niewiadomska-Bugaj,

\footnotetext{
* Correspondence: tandrews@uga.edu

${ }^{2}$ Department of Genetics, University of Georgia, Athens, GA 30602, USA Full list of author information is available at the end of the article
}

2012) or use them ineffectively (Andrews, Leonard, Colgrove, \& Kalinowski, 2011; Dancy, Henderson, \& Turpen, 2016).

Given this reality, researchers and funding agencies have recognized the need to better understand how change occurs in STEM higher education. This has resulted in increased scholarly focus on change in STEM education (Henderson et al., 2011). This has been accompanied with (and driven by) a change in funding priorities from US national agencies. For instance, starting in 2014, the National Science Foundation's (NSF) Improving Undergraduate STEM Education (IUSE) program created a strand focused on Institutional and Community Transformation. The goal of this strand is for researchers to integrate theories and findings from education research with the aim of translating theory to practice. Studies in this strand aim to generate knowledge about how educational change happens. Furthermore, this program recognizes that achieving meaningful change in undergraduate STEM education requires changing the entire system, not just faculty thinking or behavior.

A key challenge faced by projects aiming to enact change in undergraduate STEM education is knowing what actions are likely to result in the desired outcomes 
within the targeted system. In other words, what should change agents do to try to achieve change, and what might they need to know to make change in their particular context? Recognizing this challenge, funding agencies now routinely ask grant proposers to use theory. For instance, the NSF expects proposals to the Institutional and Community Transformation track of IUSE to include "one or more theories of change to guide the proposed work" (National Science Foundation, 2019). Similarly, the Howard Hughes Medical Institute's (HHMI) Inclusive Excellence Initiative expects an institution that wins the award to "develop its theory of change within the particular context of the campus" (Howard Hughes Medical Institute, 2019). Both agencies seem to expect clear articulation of how and why the planned activities are likely to lead to the desired outcomes, and may also want proposers to draw on the existing research and theory about how change occurs. Yet this may not be apparent to their audiences. These quotes suggest that NSF and HHMI may not mean the same thing when they refer to "theory of change." This is indicative of the broader ambiguity in terminology within the discipline-based education research community. NSF appears to be referring to drawing on existing theory about how change occurs-what we will call change theory. In contrast, HHMI appears to be referring to a project-specific articulation of planned activities and how they are expected work-which is referred to as a "theory of change" in the evaluation community. This example emphasizes the need for consensus around terminology (Table 1).

The goals of this essay are to:

1. Distinguish between a theory of change (and related terms) and change theory and research

2. Describe the various roles that change theory and research can play in building and refining a theory of change for a specific project

We aspire to arrive at a common language that change agents, Discipline-Based Education Research (DBER) scholars, and funding agencies can use to communicate about theory. We also hope to provide a starting place for researchers who are new to studying change and are grappling with how to ground a change initiative existing knowledge and contribute to our collective knowledge about how to achieve change in undergraduate STEM education. This essay is not a how-to guide or a list of theories of change from other projects. We direct the reader elsewhere for examples of theories of change in STEM education (Connolly \& Seymour, 2015) and for guides about how to create a theory of change for your work (Anderson, 2005; Aspen Institute Roundtable on Community Change, 2003).

\section{Theory of change}

The historical roots of a theory of change come from the field of theory-driven evaluation, which came to prominence in the 1990's (Chen, 1990; Coryn, Noakes, Westine, \& Schröter, 2011). Theory-driven evaluation aimed to move beyond a simplistic input-output notion of evaluation and instead required that program designers explicitly state how they expected a program to work, thereby making their implicit assumptions explicit. This allows an evaluator to better understand what is being implemented and why, making clear connections between a given intervention and its outcomes. By making the underlying rationale of an initiative explicit, it can be interrogated, assessed, and revised systematically as it is being implemented (cf. Design-Based Research; Cobb, Confrey, Disessa, Lehrer, \& Schauble, 2003).

The term "theory of change" itself was popularized by Weiss, through the work of the Aspen Institute and the Roundtable on Community Change (Anderson, 2005; Weiss, 1995). Within the evaluation community, this term is often capitalized (i.e., Theory of Change), but this can cause confusion among scientists who do not think of an individual project's theory of change as akin to how they use the term theory. Given our intended audience of discipline-based education researchers, we have opted for lower case letters in this commentary. To evaluate complex community initiatives focused on social change, a theory of change was designed as a tool to help clearly articulate underlying assumptions from the offset. The process of creating the theory of change allows a team to reach consensus on its underlying assumptions, which are then codified in an explicit product (often displayed as a diagram). This product

Table 1 Distinguishing between change theory and a theory of change

\begin{tabular}{llll}
\hline Terminology & Definition & Scope & Source \\
\hline Change theory & $\begin{array}{l}\text { Framework of ideas, supported by evidence, that explains some } \\
\text { aspect of change beyond a single initiative }\end{array}$ & $\begin{array}{l}\text { Generalizable } \\
\text { beyond a single } \\
\text { initiative }\end{array}$ & $\begin{array}{l}\text { Peer-reviewed literature and books } \\
\text { relevant to change in STEM higher } \\
\text { education }\end{array}$ \\
& $\begin{array}{l}\text { A particular approach for making underlying assumptions in a } \\
\text { change project explicit, and using the desired outcomes of the } \\
\text { project as a mechanism to guide project planning, implementation, } \\
\text { and evaluation }\end{array}$ & $\begin{array}{l}\text { Single change } \\
\text { initiative }\end{array}$ & Created and refined by project team \\
\hline
\end{tabular}


accounts for the context of a specific initiative. Rather than simply asking "does it work?" the goal of this process is to work toward understanding "under what conditions does something work, and for whom?" (Pawson \& Tilley, 1997). Articulating the underlying rationale for a project is not just a matter of appeasing funding agencies, but is a critical component of engaging in high-quality scholarship. Thus, many change agents are faced with the challenge of articulating a theory of change for their work. The initial theory of change for a project is really a series of hypotheses about how change will occur and these hypotheses are investigated and revised as the project proceeds. Ongoing projects constantly reconsider and revise their theory of change as they gather data that indicates whether and how their efforts are working.

Given its roots in program evaluation and social change, the methodology behind a theory of change may be less familiar to DBER scholars. However, a theory of change has similarities to many methodologies used by DBER scholars; Design-Based (Implementation) Research (Cobb et al., 2003; Penuel, Fishman, Cheng, \& Sabelli, 2011), Improvement Science (Lewis, 2015), and Backwards Design (Wiggins \& McTighe, 2005) are all examples. Because these research threads have developed largely in parallel to research from the evaluation community, they are similar to, but not connected to the scholarship around theory of change. A theory of change is a particular approach for making underlying assumptions explicit, and using the desired outcomes of a project as a mechanism to guide planning, implementation, and evaluation. We now outline the specific aspects of a theory of change with a DBER context in mind.

\section{Anatomy of a theory of change}

A comprehensive description of the elements of a theory of change was developed through the Aspen Institute Roundtable on Community Change (Anderson, 2005; Weiss, 1995). Here, we draw from the terminology used in the guidebook, recognizing it as one of the definitive sources for developing a theory of change. Because terminology is not used consistently throughout the literature, we carefully define the main terms used when talking about a theory of change. We are not advocating that what we describe below is the only approach to developing a theory of change. Rather, what we describe is based on a well-established approach from the evaluation literature and serves as a useful framework for clarifying the differences between a theory of change and change theory. In later sections, we elaborate the meaning of each component of a theory of change and discuss how change theory and research relate to these specific components of the theory of change.
The process of developing a theory of change begins with recognizing the context in which the change effort will occur. Change in STEM higher education occurs within a complex system and attending to various parts of the system helps change agents consider factors that may influence the way in which a change initiative plays out. These factors are moderators of the change process, potentially speeding, slowing, initiating, or stalling change. Since every context is different, we must understand the conditions under which something works, and how it might need to be adapted to new situations. This is only possible when a project is explicit about its particular circumstances.

After describing the context, the team goes through a process of backwards mapping, first focusing on the end results and then working backwards to describe how one is supposed to get to those results. To do so, a team makes explicit the outcomes it is trying to achieve. In general, this is done by articulating a guiding long-term outcome for the initiative. The long-term outcome will eventually be attained only after a number of mediumterm and short-term outcomes-which are called preconditions-are first achieved. Articulating the preconditions that may need to be achieved en route to the ultimate outcome makes it easier for a team to track its progress and see whether an initiative is on the right track as it unfolds. Rationales describe why particular preconditions are necessary and sufficient to achieve the long-term outcome, and how particular interventions will achieve preconditions.

A theory of change articulates the specific interventions that will be used to try to achieve preconditions and long-term outcomes. These represent the project's concrete activities. Developing well-articulated outcomes and preconditions helps a team choose interventions intentionally, as compared to a project that begins by planning its interventions and then stipulating what it hopes to achieve.

Each long-term outcome or precondition is paired with a number of indicators, which describe the types of evidence needed to determine whether or not an outcome has been achieved. These indicators may be measured in a variety of ways, depending on the research methodologies adopted by a project.

Finally, a project team articulates the assumptions behind the above elements and the linkages between them. These assumptions should be based in the prior experience of the project team and the research literature. Nonetheless, because our collective knowledge of how change occurs in STEM higher education is incomplete, teams will still rely on some assumptions when they design a project.

To depict a theory of change visually, a pathway of change is created as a visual representation for the 
connections between preconditions, long-term outcome, indicators, and interventions, and of the underlying context (Fig. 1). The lines in a pathway of change represent the hypothesized rationale of the project, and may be written out in detail alongside the project's context, in a document accompanying the diagram. Assumptions may also be listed on the pathway of change diagram. The construction of such a diagram typically begins from the top down, with a user first identifying the long-term outcome, then the preconditions on the way to the outcome, and finally appropriate interventions. These may be adjusted in light of the assumptions and context. Lastly, indicators are added.

\section{Logic models, logframes, and outcome maps}

One area of confusion for researchers is the myriad terms and representations for the underlying logic of a project. One of the most common representations is a Logic Model, which explicates, in detail, the resources that go into a project, the activities undertaken to produce the outcomes, and the tangible results of the activities (W.K. Kellogg Foundation, 2004). Logic models often communicate the overall vision or aspiration of the project, as well as short, intermediate, and long-term outcomes anticipated as a result of the project. Some theory of change advocates make a clear distinction between a theory of change and Logic Model, arguing that a theory of change is much more flexible and has more explanatory power, because a Logic Model does not always make explicit the underlying assumptions about how change will happen (e.g., Clark \& Anderson, 2004). Yet in practice, the distinction between a theory of change and Logic Model is often murky. For example, a Logic Model may explicitly articulate assumptions and relevant contextual factors of the project, explaining both the how and why of a project, similar to a theory of change (University of Kansas Center for Community Health and Development, 2018). From this perspective, a Logic Model can be conceptualized as a different (albeit more structured) format for organizing the same information that would be found in a pathway of change diagram for a theory of change. The simpler, more rigid organizational structure of a Logic Model can be beneficial for a quick summary and more easily communicating with funders and other stakeholders.

In addition to a Logic Model, a wide variety of other related terms are used. These include Program Roadmaps, Theory of Cause, Theory of Action, Concept(ual) Maps, Outcome Maps, and Logical Frameworks or LogFrames (Center for Disease Control, 2018). While there are distinctions between each of these tools, in general we view them as mechanisms for articulating and depicting a program's desired outcomes and rationale for achieving them. In this way, they can be viewed as tools that are consistent with, or at least complementary to, a theory of change. Regardless of the

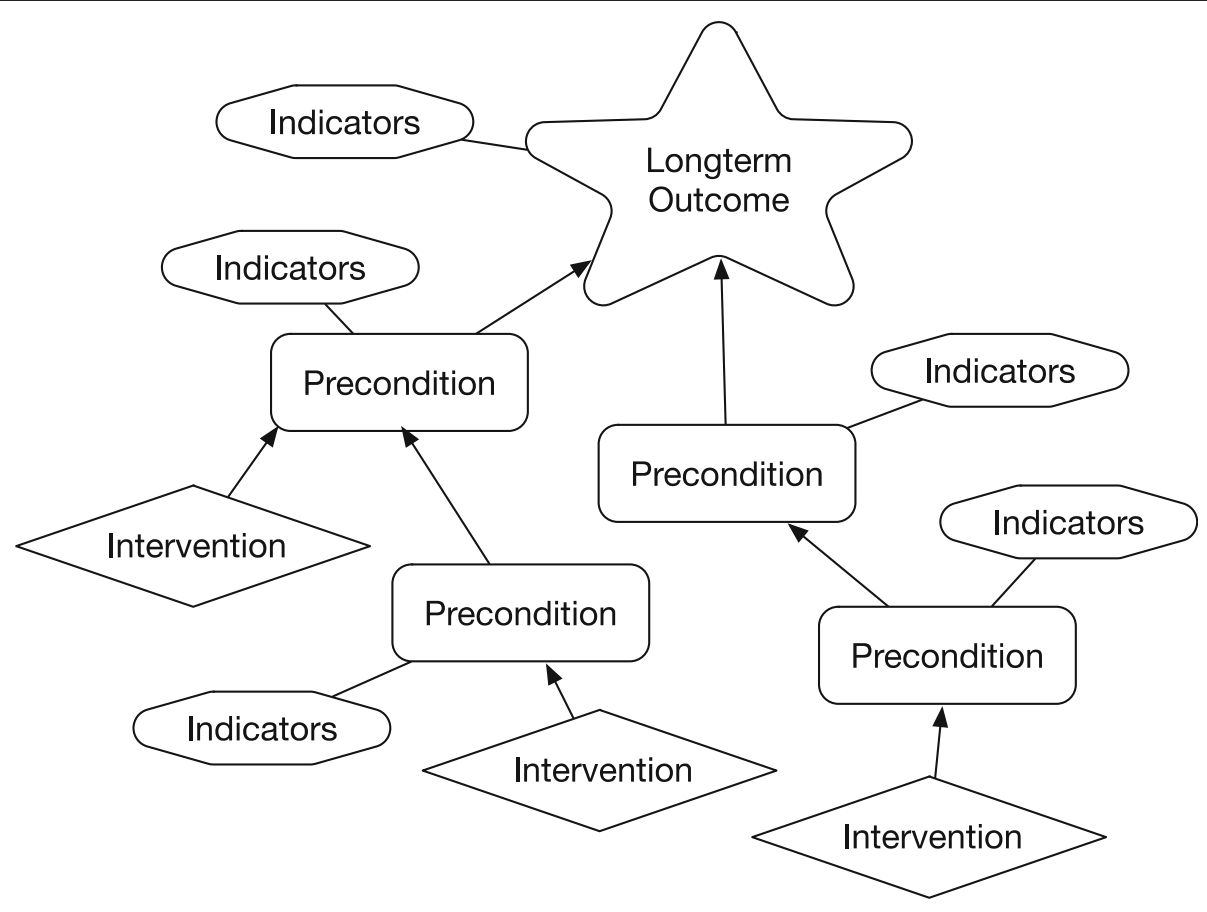

Assumptions: Assumption1, Assumption2, Assumption3

Fig. 1 Sample theory of change diagram (pathway of change) 
particular representation used, the theory guiding a particular organization or initiative should be informed by existing theory and research related to the desired outcomes.

\section{The role of change theory and research}

We define change research broadly, as any scholarship that focuses on how to make change happen. Some change research has a strong theoretical or empirical basis, while other scholarship may be more conjectural. An important subset of change research is change theory, which we define as a framework of ideas, supported by evidence, that explains some aspect of change beyond a single project.

Change theories represent generalized knowledge about how change works. Some theories are mature, with a strong empirical basis, whereas other theories may have emerged from a single context and thus are supported by more limited evidence. In the realm of educational change, there are relevant theories that deal with many different components and processes related to change.

A theory of change for a change effort in undergraduate STEM education can better serve a project and the larger community when it is developed in consultation with theory and research from the scholarly literature. The degree to which theories of change draw on change theory and research varies considerably. Change initiatives that are not informed by change research (including theory) have two major limitations: (1) the initiative is less likely to succeed, because it does not leverage the wealth of knowledge already developed by change researchers, and (2) without building on what is already known in the field, the initiative itself is less likely to contribute to generalizable knowledge, because it will be more difficult to cast the findings of the study in terms of existing scholarship.

Although there are many ways that research and theory can contribute to a change effort, we focus on five that we argue are a useful guiding framework for grounding a theory of change in existing scholarly work.
For each category, we give two examples of change research and theory to illustrate how they can inform the development of a theory of change (Table 2). The change theories and research we have used to illustrate each category do not represent endorsements of the utility or value of these particular theories, nor a negative evaluation of theories not mentioned. Given the complexity of real change scenarios, efforts can benefit from drawing on multiple change theories and diverse research areas (Kezar \& Holcombe, 2019). A single change theory can contribute to multiple aspects of a theory of change, or it can only contribute to one. Similarly, a project may draw from multiple change theories to inform each part of a theory of change. Each project is unique, which means that existing research and theory must be adapted to the specific initiative. Once a theory of change is created using an amalgam of prior work, it will represent a synthesis of change theory and research that is relevant and unique to a given project.

\section{Context}

An important advancement for education reform efforts in undergraduate STEM has been increased focus on the system and culture in which teaching and learning occur (e.g., Henderson et al., 2011). Change theory and research can inform our understanding of the context of a change effort, including relevant communities, actors, and stakeholders; existing policies, practices, and beliefs; capacity and receptiveness of the targets of the change effort; and historical, political, and sociocultural factors. Viewing change as occurring within a complex system is important because context will influence the impact of a change intervention. Theories relevant to context help change agents and researchers identify and characterize aspects of the system and culture and describe how these might moderate how a change intervention proceeds. Projects benefit from drawing on diverse research and theory to adequately describe context. To illustrate how theory can inform the recognition of context in a theory of change, we provide examples from two

Table 2 Components of a theory of change and examples of change theory and research that could inform them

\begin{tabular}{lll}
\hline Component of theory of change & Description & Examples of change theory and research \\
\hline Context & Identifying, describing, and relating parts of a system & $\begin{array}{l}\text { Teacher-Centered Systemic Reform } \\
\text { Four Frames }\end{array}$ \\
$\begin{array}{ll}\text { Outcomes, Preconditions, } \\
\text { Rationales }\end{array}$ & $\begin{array}{l}\text { What is to be achieved, what intermediate steps are } \\
\text { required, and why }\end{array}$ & $\begin{array}{l}\text { Theory of Planned Behavior } \\
\text { Indicators }\end{array}$ \\
& $\begin{array}{l}\text { Measurements of outcomes and preconditions to } \\
\text { determine if they are being achieved }\end{array}$ & Social Network Analysis \\
Interventions & Activities required to achieve a particular outcome & Analysis of STEM Teaching Practices \\
& Implicit knowledge about the context and how & River Model \\
Assumptions & Departmental Action Teams \\
& change works & Appreciative Inquiry \\
\end{tabular}


theoretical frameworks: the teacher-centered systemic reform model (TCSR) and the four frames model.

The TCSR (Woodbury \& Gess-Newsome, 2002) maps out the context of efforts that aim to change K-12 teachers' practices, including personal, structural, and cultural factors. The model inventories and describes parts of this system and why they can be expected to influence education reform. Though developed with K-12 contexts in mind, this framework can be translated to a higher education system. For example, The Cottrell Scholars Collaborative New Faculty Workshop is aligned with TCSR. The workshop explicitly addresses personal factors, teacher's thinking, and contextual factors in training newly hired chemistry assistant professors about evidence-based instructional practices (Stains, Pilarz, \& Chakraverty, 2015). Other researchers used TCSR as a framework for analyzing data about what influenced the use of the SCALE-UP model within one university (Enderle, Southerland, \& Grooms, 2013), demonstrating that a change theory may become useful for considering context late in a change initiative.

The four frames model of organizational change can draw attention to different aspects of the system, because it focuses specifically on culture within organizations. This model defines different lenses through which an organization's culture can be viewed (Bolman \& Deal, 2008). Specifically, the four frames model operationalizes culture as an ever-changing set of structures and underlying ways of thinking, and the resulting power relationships between individuals (Reinholz \& Apkarian, 2018). Applied to change efforts in undergraduate STEM, this model can define which cultural factors an agent will need to attend to in order to understand the success of an effort, as well as what a project might aim to change in a culture in order to support the desired long-term outcome (Reinholz \& Apkarian, 2018). These theories, and others like them, help change efforts recognize the various parts of their system that are likely to be important to a change effort. Since change agents work from one particular vantage point of the system (e.g., a faculty member in a department, a dean in a college, a professional developer in a Center for Teaching and Learning), it can be hard for individual actors to recognize how more distant parts of a system will influence change efforts.

In summary, change theory and research can inform a theory of change by mapping out and defining the components of a system and the various ways those components interact with each other that may influence (i.e., moderate) change efforts.

\section{Preconditions and rationale}

A key part of the process of developing a theory of change is identifying the short- and intermediate-term outcomes, called preconditions, that may need to be met to arrive at the ultimate long-term outcome of the change effort. The preconditions included in a theory of change should be necessary and sufficient to achieve the long-term outcome. Thus, there must be a rationale for linking a precondition to another precondition and for linking preconditions to the ultimate outcome (Anderson, 2005). Rationales explain how the expected interventions in a change effort are likely to result in meeting preconditions and achieving the long-term outcome. How does intervention A cause precondition $B$ to be met? And how does meeting precondition B help to achieve the long-term outcome? Change theory and research can help a team recognize important preconditions and why it will be necessary to achieve them on the path to the long-term outcome. Drawing on diverse work is crucial for developing a theory of change with preconditions and rationales that are based on more than our own intuitions about how to achieve change.

We introduce two theoretical frameworks that can inform preconditions and rationale in a theory of change, including one framework relevant to achieving behavioral change among individuals-the theory of planned behavior-and one framework relevant to achieving organizational change-the 4I framework of organizational learning.

The theory of planned behavior was developed to explain what shapes behaviors over which individuals have the ability to exert self-control. The theory of planned behavior stipulates that a key factor influencing behavior is an individual's intention to perform the behavior (Ajzen, 1991). In turn, intention is influenced by an individual's attitude toward the behavior, the subjective norms they perceive related to the behavior, and their perception of their own control over the behavior (Ajzen, 1991). Attitudes encompass the individual's favorable or unfavorable evaluation of the behavior. Subjective norms include perceived social pressures or expectations to perform or not perform the behavior. Perceived behavioral control has similarities to selfefficacy, and deals with an individual's confidence in their ability to perform a behavior successfully (Ajzen, 1991; Bandura, 1997).

Applied to a change effort to increase the adoption of evidence-based teaching practices, the theory of planned behavior proposes that preconditions to adopting a new teaching strategy include a positive attitude about the strategy, perceptions of social pressure to adopt the strategy, and confidence that one can successfully adopt the strategy. The theory stipulates the preconditions and provides rationales for why we can expect these preconditions to lead to the long-term outcome of the adoption of evidence-based teaching strategies. 
The 4I framework of organizational learning describes processes involved in creating, retaining, and transferring knowledge within an organization (Crossan, Lane, \& White, 1999). The framework contends that organizational learning is a multi-level process that involves individual, group, and organizational learning. Individual, group, and organization learning are connected by four bi-directional processes: intuiting, interpreting, integrating, and institutionalizing (Crossan et al., 1999). Intuiting occurs at the level of the individual and involves developing insights based on personal experience that can be "fed forward" beyond the individual. These insights might recognize patterns and new possibilities based on personal experience and may become metaphors that individuals use to talk about experiences with others. Interpreting is a bridge between individuals and groups, and involves explaining an insight to oneself and others. This level is more conscious than intuiting and involves conversation and dialogue that names ideas, makes them explicit, and links them to other ideas. This can lead to enhanced mental models among individuals and improved organizational knowledge. Integrating is the third process and it connects the group and organizational levels. It involves developing shared understanding among individuals and taking coordinated actions. This process is focused on coherent, collective action. The last process, institutionalization, occurs at the organization level when new ideas and actions become embedded into routines, rules, procedures, and infrastructures. This is the hallmark of organizational learning because changes in structures makes the change available to all members, independent of the original individual or group that generated the idea.

The Center for the Integration of Research, Teaching, and Learning (CIRTL), a network of 38 research institutions, used the 4I model of organizational learning to analyze the impact that CIRTL had on member institutions (Hill, Savoy, Austin, \& Bantawa, 2019). They observed intuiting among local institutional CIRTL leaders, interpreting in the work between local leaders and local constituents, and integration of learning gains associated with CIRTL across campus by the local leaders (Hill et al. 2019). The 4I model helped these researchers recognize the role of the local CIRTL leader in all four of the organizational learning processes in the $4 \mathrm{I}$ model. Thus, a precondition for the impact of the CIRTL network on member institutions may be a local CIRTL leadership team that can translate and tailor the network efforts to the specific needs of their institution (Hill et al., 2019).

This model could also be used to inform the design of a change project. For example, applied to an effort to change how teaching is evaluated for promotion and tenure, the 4I framework of organizational learning suggests that a precondition to institutionalizing new structures includes individuals in a group (e.g., a department) developing shared understandings. In this example change effort, that might be shared understanding about what constitutes effective teaching and how effectiveness can be documented. Shared understandings can then inform collective actions, such as piloting a new evaluation approach. Developing shared understandings occurs through interpreting and integrating, and relies heavily on developing common language, ongoing dialogue, and even storytelling to reflect complexity without abstraction (Crossan et al., 1999). These shared understandings then provide the basis for a change that can affect the organization as a whole.

In summary, change theory and research can inform a theory of change by helping a team determine what preconditions are likely to be important to achieve a desired long-term outcome and why those particular preconditions are important.

\section{Indicators}

Indicators are how a project team determines if each precondition and the long-term outcome have been met. Ideally, a theory of change specifies one or more indicators for each precondition and the long-term outcome. This allows a project to assess the degree to which implementation of project activities is having the intended impact. Indicators must be fully operationalized to usefully inform a project. Operationalizing an indicator includes determining what variable will be measured, the target population for change, what threshold of change will be sufficient to conclude that a precondition or outcome has been met, and how long it is expected to take to achieve this threshold in the target population (Anderson, 2005; Aspen Institute Roundtable on Community Change, 2003). Setting indicators aids in setting outcomes for a project, so they should be set early in the planning stages of a project. In addition, assessing indicators helps measure progress made toward those outcomes as a project is being implemented. For members of a project team, assessing indicators can help provide motivation as it makes progress towards outcomes visible. It also provides formative feedback to support revision of activities and the theory of change as a whole. Furthermore, assessment of indicators helps communicate the outcomes and progress toward outcomes for people outside of the project team (Kotter, 1996).

Many researchers have developed approaches and tools to measure important variables in the context of undergraduate STEM education. Change research draws attention to variables that can be measured and how to measure them, including a wide variety of 
qualitative and quantitative research techniques and methodologies. Projects may use surveys and inventories, interviews, systematic classroom observations, participant observation, and other approaches to collect data about an indicator.

Some desired outcomes in a change project in undergraduate STEM education may deal with teaching practices. Many possible indicators for teaching practices are described in a single report that reviews approaches to measuring and documenting STEM teaching practices (American Association for the Advancement of Science, 2012). Some projects may aim to measure the quality of education on a larger scale. The National Academies of Science, Engineering, and Medicine published a report that outlines a set of national-level indicators of the status and quality of undergraduate STEM education (National Academies of Sciences, Engineering, and Medicine, 2018).

Change projects in undergraduate STEM education will also include important preconditions and long-term outcomes besides changes in teaching practice. For example, some initiatives aim to change thinking, relationships, policies, and practices in departments, colleges, and institutions (Elrod \& Kezar, 2015; Reinholz, Corbo, Dancy, \& Finkelstein, 2017). This requires different indicators. For instance, research on social networks can be useful because it helps identify opinion leaders and tracks relationships across a social system, such as a department (e.g., Andrews, Conaway, Zhao, \& Dolan, 2016; Grunspan, Wiggins, \& Goodreau, 2014; Hayward \& Laursen, 2018; Quardokus \& Henderson, 2015). Examples of social network indicators may be increasing the number of opinion leaders, increasing the density of a network, or increasing the strength of relationships related to undergraduate teaching in a department. Another example of indicators relevant to departments are rubrics developed by the Partnership for Undergraduate Life Sciences Education (PULSE) to assess the degree to which life sciences undergraduate programs align with recommendations in the Vision \& Change Report. These rubrics can be used by departments and administrators to assess the degree to which curriculum alignment, assessment, faculty practice and support, infrastructure, and climate for change are supportive of the recommendations in Vision \& Change (AAAS, 2011; BrancaccioTaras et al., 2016).

In summary, change theory and research can inform a theory of change by helping a team determine which indicators can assess progress toward a long-term outcome and the preconditions on the way to the outcome.

\section{Interventions}

An intervention is what a project "does" to try to achieve change. Interventions are often described as a list of steps, even though the steps may be nonlinear and not all steps are always followed in order. Many different terms are used to describe interventions, such as strategy, process, approach, and model. The intervention provides an organizing framework for the types of project activities that a change effort engages in. Often, a project team has made tentative decisions about their intervention before they begin developing a theory of change. Building a theory of change ensures that careful consideration has been given to how the activities will lead to preconditions and how preconditions contribute to achieving the long-term outcome.

Change interventions are generally part of the change research literature, rather than being change theories themselves. Some interventions that have been implemented and studied are solidly grounded in existing change theory and others have less robust theoretical underpinnings. Which change interventions have been designed and revised with change theory in mind may become obvious as a team builds their own theory of change around an intervention and is faced with questions about how the suggested activities will result in the desired outcomes. Here, we highlight two change interventions designed specifically for STEM higher education and grounded in existing change theory: the Keck/PKAL model (i.e., River Model) and Departmental Action Teams.

The Keck/PKAL (Project Kaleidoscope) model, sometimes referred to as the River Model, is a process for achieving institutional change in undergraduate STEM education and its steps are meant to be spearheaded by campus leaders (Elrod \& Kezar, 2015). This model is informed by change theories about organizational learning, organizational culture, readiness for change, and more. The River Model includes eight stages: (1) establishing vision, (2) examining the landscape and conducting capacity analysis, (3) identifying and analyzing challenges and opportunities, (4) choosing strategies, (5) determining readiness for action, (6) beginning implementation, (7) measuring results, and (8) disseminating results and planning next steps.

Some of these steps are expected to occur in a loop and through multiple iterations, resulting in the change project being "caught in an eddie" as team members work toward shared understandings and consensus. For example, stages (1) establishing vision and (2) examining the landscape and conducting capacity analysis are envisioned as two looping and iterative processes that ultimately align a change effort with institutional priorities. Though these two stages are the suggested starting place, the model is meant to be dynamic and the process can be entered at multiple points (Kezar \& Elrod, 2015). Additionally, projects may sometimes flow "upstream" rather than "downstream" in the pursuit of change. 
Kezar and Elrod (2015) is a report designed to guide step-by-step planning, provide tools, and prompt ongoing reflection. A change effort that opts to use the River Model as its guiding intervention may also need to incorporate interventions at other levels of the system, such as departments and individual faculty.

Departmental Action Teams (DATs) are externally facilitated groups of faculty, students, and staff working collaboratively in a department toward a collective educational outcome. DATs focus on a single department as a unit of change, recognizing that (a) departments tend to have relatively consistent cultures, and (b) making sustainable changes to education requires cultural change (Reinholz et al., 2019). A DAT meets regularly for up to four semesters, typically for an hour every other week. To guide its work, a DAT completes a series of shared visioning activities to develop consensus on a focal issue and set of outcomes. Next, the DAT collects, analyzes, and interprets relevant data. This supports the creation and implementation of an action plan, as well as monitoring of that plan. Typically, DATs aim to create a sustainable structure within a department that leads to continuous improvement, rather than trying to "solve" a problem all at once.

To utilize DATs a part of a larger change initiative, a project team would need to hire or develop skilled external facilitators who could lead DATs in academic departments. The use of DATs would also inform the overall approach of a project. Because DATs focus on lasting, sustainable changes, they would be most appropriate for a multi-year effort that aims for cross-cutting improvements. For example, if a project team were to apply DATs to achieve an outcome of improving instruction in a department, potential outcomes of the DATs would be (1) onboarding and mentoring opportunities for new faculty; (2) developing mechanisms for sustained, discipline-based professional development in the department; or (3) seminars, colloquium, and brown bag meetings for faculty to talk about teaching. All of these outcomes would be aimed at supporting teaching over the long-term.

A single change initiative may draw upon multiple interventions to achieve its outcomes. There is not a single best intervention, and very often, different interventions could play a similar role in a project. Thus, in building a theory of change, a team may identify multiple interventions that can be used and adapted to the particular context of interest.

\section{Assumptions}

A major feature of a theory of change is the articulation of underlying assumptions about how change occurs. These include assumptions about the nature of the context and how it will moderate the change process, the preconditions that are necessary and sufficient to reach the long-term outcome, the rationales connecting preconditions to each other and to long-term outcomes, the rationales for how interventions will achieve preconditions and the long-term outcome, and more. Identifying and articulating assumptions is challenging because they may be taken for granted and deeply held (Argyris, 1976). Challenging assumptions is likely to occur throughout a change project, as some assumptions may only come to light when aspects of an initiative fail. Thus, change research and theory should inform the initial development of a theory of change. Critically, it is common for change efforts to fail in various ways. Change theory can be very useful for guiding sensemaking and trouble-shooting that takes place when aspects of the project are stalled or unsuccessful.

Given that assumptions may be found throughout a theory of change, diverse change theory and research will be relevant to articulating assumptions. Here, we highlight two lenses that propose alternative ways of thinking about areas relevant to change in undergraduate STEM education: community cultural wealth and appreciative inquiry. These two lenses advocate for moving away from deficit views focused on what is lacking or problematic toward views focused on existing and diverse strengths and successes.

Community cultural wealth is a framework that characterizes "knowledge, skills, abilities, and contacts possessed and utilized by communities of color to survive and resist macro- and micro-forms of oppression" (Yosso, 2005). This framework recognizes different forms of capital that are resources that people of color develop and use and that contribute to their success. For example, linguistic capital includes cognitive and social skills gained through experiences communicating in more than one language or style (Yosso, 2005, Means et al., in review).

Community cultural wealth can be a useful lens for change efforts related to broadening participation in STEM. For example, using this lens, an effort to increase the hiring and retention of faculty from groups minoritized in STEM may recognize that their planned activities are grounded in deficit thinking because they aim to support new faculty in areas in which they are assumed to be lacking, rather than capitalizing on the unique strengths and capital of these new hires. Adopting such a framework, a hiring committee may aim to see how a new hire would add to their culture, rather than just fitting in it.

Appreciative inquiry is a stance toward organizational change that assumes that organizations have infinite constructive capacity to improve. Appreciative inquiry achieves change by discovering, magnifying, and eclipsing positive attributes and successes in an organization, 
rather than focusing on identifying and solving problems (Cooperrider \& Whitney, 2001). This stance on change translates into a change process that asks questions of many (or all) members of an organization to learn what is working very well in an organization. A basic principle of appreciative inquiry is that organizations change in areas where they persistently ask questions, and the more positive the questions asked, the more long-lasting and successful a change effort will be (Cooperrider \& Whitney, 2001).

Using appreciative inquiry as a lens to examine a change effort may reveal contrasting or contradictory assumptions like these: the outcome of a change effort is to solve problems; members of the organization are not a valuable source of ideas about what or how to change; and easily measured successes are more important to the momentum of an effort than feelings like hope, excitement, inspiration, caring, and joy (Cooperrider \& Whitney, 2001).

Change research can also reveal other assumptions that may be implicit in a theory of change. For example, it is tempting to conclude that scientists will be convinced to adopt evidence-based teaching practices when presented with strong evidence of the effectiveness of the strategies. However, research on why faculty adopt evidence-based teaching strategies indicate that they rely on personal experiences to a much greater extent than empirical evidence (Andrews \& Lemons, 2015; Dancy et al., 2016). Thus, a theory of change that included presenting faculty with evidence to increase their interest and motivation to use evidence-based practices should reconsider assumptions they are making about how faculty use evidence in making teaching decisions. As another example, researchers might assume that lasting changes can be made through a single, momentous change event, rather than through careful, ongoing attention to sustainable improvements. However, research indicates that even very successful change efforts may ultimately result in backsliding, unless explicit attention is paid to sustainability (Reinholz et al., 2019).

In summary, all change efforts are built around some assumptions, but the construction of a theory of change helps make those assumptions explicit, and can ensure that the assumptions are grounded in prior research and practice as much as possible.

\section{Summary and conclusion}

This essay sought to distinguish between change theory and research and a theory of change in STEM education. Ultimately, we argue that change efforts should develop their own theory of change that is grounded in change theory. In this way, a project contextualizes change theory to their own particular situation, which will increase the likelihood of project's success, and also enhances the project's capacity to contribute to generalizable knowledge. Far from exhaustive, this manuscript provides examples of how some commonly used change theories can inform a change project. We also propose common terminology that DBER communities could use to refer to the articulated logic of an individual project (Tables 1 and 2).

Given that STEM educational change research is conducted by a diverse community of researchers and practitioners across disciplines, it is often difficult to compare across studies. A theory of change can help to address this challenge. As teams make their underlying assumptions and theories explicit, it will facilitate comparisons of research across diverse settings. In addition, when theory is set from the offset, the implementation of a project provides a test of the theory in practice, which supports refinement of the theory. We propose a theory of change as a powerful mechanism for making theory explicit both for a project internally and for externalization.

\section{Abbreviations}

DAT: Departmental Action Team; DBER: Discipline-based education research; HHMI: Howard Hughes Medical Institute; IUSE: Improving Undergraduate STEM Education; NSF: National Science Foundation; PKAL: Project Kaleidoscope; STEM: Science, technology, engineering, mathematics; TCSR: Teacher-centered systemic reform

\section{Acknowledgments}

The authors are grateful for ongoing efforts to build community around the scholarship of change in STEM education. The authors began their collaboration after meeting at the Transforming Research in Undergraduate STEM Education (TRUSE) Conference. We have also benefitted from the support of the Accelerating System Change Network (ASCN) and the STEMDBER Alliance. We thank Alice Olmstead, Erika Offerdahl, Isabel White, and Susan Shadle for their comments on earlier drafts of this paper. We also thank two anonymous reviewers for feedback that improved the quality of this manuscript.

Author's contributions

DLR and TCA collaborated to conceive of these ideas and write the manuscript. Both authors read and approved the final manuscript.

\section{Funding}

Partial support for this work was provided by the National Science Foundation's Improving Undergraduate STEM (IUSE) program under awards 1830897 and 1830860. Any opinions, findings, and conclusions or recommendations expressed in this material are those of the authors and do not necessarily reflect the views of the National Science Foundation.

Availability of data and materials Not applicable.

\section{Competing interests}

The authors declare that they have no competing interest.

\section{Author details}

'Department of Mathematics and Statistics, San Diego State University, San Diego, CA 92182, USA. ²Department of Genetics, University of Georgia, Athens, GA 30602, USA. 
Received: 25 September 2019 Accepted: 2 January 2020

\section{Published online: 17 January 2020}

\section{References}

Ajzen, I. (1991). The theory of planned behavior. Organizational Behavior and Human Decision Processes, 50(2), 179-211. https://doi.org/10.1016/07495978(91)90020-T

American Association for the Advancement of Science. (2011). Vision and Change in Undergraduate Biology Education: A call to action. Washington, D.C.: American Association for the Advancement of Science.

American Association for the Advancement of Science. (2012). Describing \& Measuring Undergraduate STEM Teaching Practices. Retrieved from American Association for the Advancement of Science website: https://liveccliconference.pantheonsite.io/measuring-teaching-practices/ Accessed 12 Sept 2019.

Anderson, A. (2005). The community builder's approach to theory of change: A practical guide to theory development. Retrieved from Aspen Institute Roundtable on Community Change website: http://www.theoryofchange. org/pdf/TOC_fac_guide.pdf.

Andrews, T. C., Conaway, E. P., Zhao, J., \& Dolan, E. L. (2016). Colleagues as change agents for undergraduate teaching. CBE-Life Sciences Education, 15(2), 1-17. https://doi.org/10.1187/cbe.15-08-0170.

Andrews, T. C., \& Lemons, P. P. (2015). It's personal: Biology instructors prioritize personal evidence over empirical evidence in teaching decisions. CBE-Life Sciences Education, 14(1), ar7.

Andrews, T. M., Leonard, M. J., Colgrove, C. A., \& Kalinowski, S. T. (2011). Active learning not associated with student learning in a random sample of college biology courses. CBE_Life Sciences Education, 10(4), 394-405. https://doi.org/ 10.1187/cbe.11-07-0061.

Argyris, C. (1976). Single-loop and double-loop models in research on decision making. Administrative Science Quarterly, 363-375.

Aspen Institute Roundtable on Community Change. (2003). Guided example: Project Superwoman. Retrieved from https://www.theoryofchange.org/pdf/ Superwomen_Example.pdf.

Bandura, A. (1997). Self-efficacy: The exercise of control. New York: Freeman.

Bolman, L. G., \& Deal, T. E. (2008). Reframing organizations: Artistry, choice, and leadership. San Francisco, CA: Jossey-Bass.

Brancaccio-Taras, L., Pape-Lindstrom, P., Peteroy-Kelly, M., Aguirre, K., AwongTaylor, J., Balser, T., et al. (2016). The PULSE vision \& change rubrics, Version 1. 0 : A valid and equitable tool to measure transformation of life sciences departments at all institution types. CBE-Life Sciences Education, 15(4), ar60 https://doi.org/10.1187/cbe.15-12-0260.

Center for Disease Control. (2018). Evaluation guide: Developing and using a logic model. Retrieved from https://www.cdc.gov/eval/logicmodels/index.htm.

Chasteen, S., Chattergoon, R., Prather, E., \& Hilborn, R. (2016, July 20-21). Evaluation methodology and results for the new faculty workshops. Paper presented at Physics Education Research Conference 2016, Sacramento, CA. Retrieved from http://www.compadre.org/Repository/document/ServeFile. $\mathrm{cfm}$ ? $\mathrm{D}=14196 \& \mathrm{Doc} \mid \mathrm{D}=4548$.

Chen, H. T. (1990). Theory-driven evaluations. Thousand Oaks, CA: Sage

Clark, H., \& Anderson, A. A. (2004). Theories of change and logic models: Telling them apart. Annual Meeting of the American Evaluation Association. Presented at Atlanta, GA.

Cobb, P., Confrey, J., Disessa, A., Lehrer, R., \& Schauble, L. (2003). Design experiments in educational research. Educational Researcher, 32(1), 9-13. https://doi.org/10.3102/0013189X032001009.

Connolly, M. R., \& Seymour, E. (2015). Why theories of change matter (No. WCER Working Paper No. 2015-2). Retrieved from Wisconsin Center for Education Research website: https://wcer.wisc.edu/docs/working-papers/Working_ Paper_No_2015_02.pdf.

Cooperrider, D., \& Whitney, D. (2001). A positive revolution in change: Appreciative inquiry. Public Administration and Public Policy, 87, 611-630.

Coryn, C. L. S., Noakes, L. A., Westine, C. D., \& Schröter, D. C. (2011). A systematic review of theory-driven evaluation practice from 1990 to 2009. American Journal of Evaluation, 32(2), 199-226. https://doi.org/10.1177/ 1098214010389321

Crossan, M. M., Lane, H. W., \& White, R. E. (1999). An organizational learning framework: From intuition to institution. The Academy of Management Review, 24(3), 522-537. https://doi.org/10.2307/259140.

Dancy, M., Henderson, C., \& Turpen, C. (2016). How faculty learn about and implement research-based instructional strategies: The case of peer instruction. Physical Review Physics Education Research, 12(1), 010110. https:// doi.org/10.1103/PhysRevPhysEducRes.12.010110.

Derting, T. L., Ebert-May, D., Henkel, T. P., Maher, J. M., Arnold, B., \& Passmore, H. A. (2016). Assessing faculty professional development in STEM higher education: Sustainability of outcomes. Science Advances, 2(3), e1501422.

Ebert-May, D., Derting, T. L., Henkel, T. P., Middlemis Maher, J., Momsen, J. L., Arnold, B., \& Passmore, H. A. (2015). Breaking the cycle: future faculty begin teaching with learner-centered strategies after professional development. CBE_Life Sciences Education, 14(2), ar22.

Ebert-May, D., Derting, T. L., Hodder, J., Momsen, J. L., Long, T. M., \& Jardeleza, S. E. (2011). What we say is not what we do: Effective evaluation of faculty professional development programs. Bioscience, 61(7), 550-558.

Elrod, S., \& Kezar, A. (2015). Increasing student success in STEM: A guide to systemic institutional change. A Keck/PKAL Project at the Association of American Colleges \& Universities.

Enderle, P. J., Southerland, S. A., \& Grooms, J. A. (2013). Exploring the context of change: Understanding the kinetics of a studio physics implementation effort. Physical Review Special Topics-Physics Education Research, 9(1), 010114

Grunspan, D. Z., Wiggins, B. L., \& Goodreau, S. M. (2014). Understanding classrooms through social network analysis: a primer for social network analysis in education research. CBE-Life Sciences Education, 13(2), 167-178. https://doi.org/10.1187/cbe.13-08-0162.

Hayward, C. N., \& Laursen, S. L. (2018). Supporting instructional change in mathematics: using social network analysis to understand online support processes following professional development workshops. International Journal of STEM Education, 5(1), 28. https://doi.org/10.1186/s40594-018-0120-9.

Henderson, C. (2008). Promoting instructional change in new faculty: An evaluation of the physics and astronomy new faculty workshop. American Journal of Physics, 76(2), 179-187.

Henderson, C., Beach, A., \& Finkelstein, N. (2011). Facilitating change in undergraduate STEM instructional practices: an analytic review of the literature. Journal of Research in Science Teaching, 48(8), 952-984.

Henderson, C., Dancy, M., \& Niewiadomska-Bugaj, M. (2012). Use of researchbased instructional strategies in introductory physics: where do faculty leave the innovation-decision process? Physical Review Special Topics Physics Education Research, 8(2), 020104. https://doi.org/10.1103/ PhysRevSTPER.8.020104.

Howard Hughes Medical Institute. (2019). Inclusive excellence new competition announcement. Retrieved from https://www.hhmi.org/science-education/ programs/inclusive-excellence-new-competition-announcement. .

Kezar, A. J., \& Holcombe, E. M. (2019). Leveraging multiple theories of change to promote reform: an examination of the AAU STEM initiative. Educational Policy, 0895904819843594. https://doi.org/10.1177/ 0895904819843594.

Kotter, J. P. (1996). Leading change. Boston, MA: Harvard Business Review Press.

Lewis, C. (2015). What is improvement science? Do we need it in education? Educational Researcher, 44(1), 54-61. https://doi.org/10.3102/ $0013189 \times 15570388$.

Means, D., Stanton, J., Mekonnen, B., Oni, O., Breeden, R., Babatola, O., Osondu, C., Beckham, M., \& Marshall, B. A deeper calling: the aspirations and persistence of black undergraduate students in Science. American Education Research Journal in review.

National Academies of Sciences, Engineering, and Medicine. (2018). Indicators for Monitoring Undergraduate STEM Education. Retrieved from The National Academies Press website: doi: https://doi.org/10.17226/24943

National Science Foundation. (2019). Improving Undergraduate STEM Education: Education and Human Resources (IUSE: EHR). Retrieved from https://www. nsf.gov/publications/pub_summ.jsp?ods_key=nsf19601.

Olmstead, A., \& Turpen, C. (2016). Assessing the interactivity and prescriptiveness of faculty professional development workshops: The real-time professional development observation tool. Physical Review Physics Education Research, 12(2), 020136

Pawson, R., \& Tilley, N. (1997). Realistic evaluation. London, UK: Sage.

Penuel, W. R., Fishman, B. J., Cheng, B. H., \& Sabelli, N. (2011). Organizing research and development at the intersection of learning, implementation, and design. Educational Researcher, 40(7), 331-337.

Quardokus, K., \& Henderson, C. (2015). Promoting instructional change: Using social network analysis to understand the informal structure of academic departments. Higher Education, 70(3), 315-335. https://doi.org/10.1007/ s10734-014-9831-0. 
Reinholz, D. L., \& Apkarian, N. (2018). Four frames for systemic change in STEM departments. International Journal of STEM Education, 5(1), 3. https://doi.org/ 10.1186/s40594-018-0103-x.

Reinholz, D. L., Corbo, J. C., Dancy, M., \& Finkelstein, N. (2017). Departmental Action Teams: Supporting faculty learning through departmental change. Learning Communities Journal, 9, 5-32.

Reinholz, D. L., Ngai, C., Quan, G., Pilgrim, M. E., Corbo, J. C., \& Finkelstein, N. (2019). Fostering sustainable improvements in science education: An analysis through four frames. Science Education. https://doi.org/10.1002/sce.21526.

Stains, M., Harshman, J., Barker, M. K., Chasteen, S. V., Cole, R., DeChenne-Peters, S. E., et al. (2018). Anatomy of STEM teaching in North American universities. Science, 359(6383), 1468-1470.

Stains, M., Pilarz, M., \& Chakraverty, D. (2015). Short and long-term impacts of the Cottrell Scholars collaborative new faculty workshop. Journal of Chemical Education, 92(9), 1466-1476.

University of Kansas Center for Community Health and Development. (2018). Community Toolbox: Developing a logic model or theory of change. Retrieved from https://ctb.ku.edu/en/table-of-contents/overview/models-forcommunity-health-and-development/logic-model-development/main. Accessed 20 April 202019.

W.K. Kellogg Foundation. (2004). Logic model development guide: Using logic models to bring together planning, evaluation, \& action. Battle Creek, Ml: W. K. Kellogg Foundation.

Weiss, C. H. (1995). Nothing as practical as good theory: Exploring theory-based evaluation for comprehensive community initiatives for children and families. New Approaches to Evaluating Community Initiatives: Concepts, Methods, and Contexts, 1, 65-92.

Wiggins, G. P., \& McTighe, J. (2005). Understanding by design. Alexandria, VA: Association for Supervision and Curriculum Development.

Woodbury, S., \& Gess-Newsome, J. (2002). Overcoming the paradox of change without difference: A model of change in the arena of fundamental school reform. Educational Policy, 16(5), 763-782. https://doi.org/10.1177/ 089590402237312.

Yosso, T. J. (2005). Whose culture has capital? A critical race theory discussion of community cultural wealth. Race Ethnicity and Education, 8(1), 69-91. https:// doi.org/10.1080/1361332052000341006

\section{Publisher's Note}

Springer Nature remains neutral with regard to jurisdictional claims in published maps and institutional affiliations.

\section{Submit your manuscript to a SpringerOpen ${ }^{\circ}$ journal and benefit from:}

- Convenient online submission

- Rigorous peer review

- Open access: articles freely available online

- High visibility within the field

- Retaining the copyright to your article

Submit your next manuscript at $\boldsymbol{\nabla}$ springeropen.com 